\title{
Uso de servicios de salud y enfermedades transmisibles de la población migrante y refugiada venezolana
}

\section{Use of health services and communicable diseases of the Venezuelan migrant and refugee population}

\author{
Johan-Sebastián Ariza-Abril' ${ }^{1}$; Ángela Marcela-Cifuentes ${ }^{1}$; \\ Mariana Calderón-Jaramillo ${ }^{1}$; Danny Rivera ${ }^{1}$; Juan-Carlos Rivillas-García ${ }^{1}$
}

Forma de citar: Ariza Abril JS, Cifuentes AM, Calderón Jaramillo M, Rivera D, Rivillas García JC. Uso de servicios de salud y enfermedades transmisibles de la población migrante y refugiada venezolana. Salud UIS. 2020; 52(4): $392-401$. doi: https://doi.org/10.18273/revsal.v52n4-2020006 (c) (i)

\section{Resumen}

Introducción: Colombia es el principal país receptor de población migrante venezolana. Esto ha implicado identificar las necesidades en salud de esta población, como la atención y tratamiento de enfermedades infecciosas. Objetivo: analizar el uso de servicios de salud relacionados con VIH/SIDA, malaria y hepatitis virales en migrantes y refugiados venezolanos en Colombia durante 2018 y 2019. Metodología: estudio de investigación mixto. Se desarrollaron 12 grupos focales con mujeres y hombres venezolanos y se realizó un análisis del uso de servicios de salud por diagnósticos de VIH/SIDA, malaria y hepatitis virales, durante 2018 y 2019, en seis ciudades con un alto flujo de migración: Barranquilla, Bogotá, Cartagena, Cúcuta, Santa Marta y Riohacha. Resultados: el uso de servicios de salud por enfermedades infecciosas en migrantes venezolanos aumentó de $2018(\mathrm{n}=1,519)$ a 2019 $(n=3,988)$. Los hombres fueron los que más usaron los servicios de salud por estas enfermedades. Aun así, la situación irregular migratoria, deficiencias en la atención primaria y acceso limitado a protección y detección temprana de enfermedades transmisibles, constituyen las principales barreras para migrantes y refugiados respecto a la atención en salud. Conclusiones: la migración expone a las personas al riesgo de contraer enfermedades infecciosas, así como a desigualdades y exclusión social en el acceso a servicios de salud para el control y tratamiento de estas enfermedades. Por ello, en contextos de migración se debe fortalecer la equidad en los servicios de salud con el fin de asegurar el acceso de las personas a la atención primaria, insumos, pruebas diagnósticas y tratamiento de enfermedades infecciosas.

Palabras clave: Migración humana; Enfermedades transmisibles; Malaria; VIH; Hepatitis viral humana; Servicios de salud.

1. Asociación Profamilia. Bogotá D.C., Colombia.

Correspondencia: Juan Carlos Rivillas. Dirección: Calle 34 14-52. Asociación Profamilia Bogotá. Teléfono: +573147105369 . Correo electrónico: juan.rivillas@profamilia.org.co 


\begin{abstract}
Introduction: Colombia is the main receiving country of the Venezuelan migrant population. This has involved identifying the health needs of this population, such as the care and treatment of infectious diseases. Objective: To analyze the use of health services related to HIV/AIDS, malaria and viral hepatitis in Venezuelan migrants and refugees in Colombia during 2018 and 2019. Methodology: Mixed research study. Twelve focus groups were held with Venezuelan women and men and an analysis was made of the use of health services for HIV/AIDS, malaria, and viral hepatitis diagnoses during 2018 and 2019 in six cities with a high migration flow: Barranquilla, Bogotá, Cartagena, Cúcuta, Santa Marta, and Riohacha. Results: The use of health services for infectious diseases among Venezuelan migrants increased from $2018(n=1.519)$ to $2019(n=3.988)$. Men were the greatest users of health services for these diseases. Even so, irregular migration status, deficiencies in primary care, and limited access to protection and early detection of communicable diseases are the main barriers to health care for migrants and refugees. Conclusions: migration exposes people to the risk of infectious diseases, as well as to inequalities and social exclusion in access to health services for the control and treatment of these diseases. Therefore, in migration contexts, equity in health services should be strengthened by ensuring people's access to primary care, inputs, diagnostic tests and treatment of infectious diseases.
\end{abstract}

Keywords: Human migration; Communicable diseases; Malaria; HIV; Human viral hepatitis; Health services.

\section{Introducción}

La migración es un determinante estructural de la salud, ya que pone en riesgo a las personas durante los flujos migratorios, pues son más propensas a experimentar exclusión social en el uso de servicios de salud; y están expuestas a un mayor riesgo de contraer enfermedades infecciosas ${ }^{1-3}$. El vínculo entre migración y salud es complejo, pues el antes, durante y después de la movilización tiene múltiples afectaciones sobre el estado de salud de la población migrante ${ }^{4,5}$. En particular, los migrantes en situación irregular, y quienes aún no legalizan su estatus migratorio en los países de acogida, son los más excluidos por los servicios de salud ${ }^{2}$.

Las enfermedades infecciosas dentro de contextos migratorios pueden ser adquiridas durante el tránsito de un lugar a otro 3,4 ; sin embargo, es difícil estimar indicadores de prevalencia de estas enfermedades en dichos contextos por la alta movilidad y por su costo $^{1}$. Por lo tanto, no resulta sencillo diferenciar si las enfermedades infecciosas fueron adquiridas por los migrantes en su país de origen, durante el tránsito o en el país receptor.

En Colombia, según el último reporte del Grupo Interangencial de Flujos Migratorios Mixtos (GIFMM), 1.8 millones de migrantes venezolanos se encuentran en diferentes regiones del país. Según su estatus migratorio, 719189 migrantes son regulares y 911.714 son irregulares; el $52 \%$ son hombres y el $48 \%$ son mujeres, además, se estima que 4.3 millones de venezolanos son migrantes pendulares ${ }^{5}$.
Gran parte de las personas migrantes venezolanas que se encuentran en Colombia viven en condiciones de vulnerabilidad, y solo el $15 \%$ de los migrantes en condición regular se ha afiliado al Sistema General de Seguridad Social en Salud (SGSSS) ${ }^{6}$. De acuerdo con la sentencia unificada 677 del 2017 de la Corte Constitucional, todos los extranjeros, incluso aquellos en condición irregular, que se encuentren en estado de vulnerabilidad tienen derecho a acceder a atención básica en salud y de urgencias ${ }^{7}$. No obstante, los migrantes pueden enfrentarse a una serie de barreras en el acceso a servicios de salud, principalmente en atención primaria, exámenes diagnósticos y medicamentos para la prevención y tratamiento de enfermedades transmisibles ${ }^{1}$; a menos que lleguen en un estado de emergencia que sustente la atención de urgencias.

Según el Ministerio de Salud y Protección Social ${ }^{8}$, las enfermedades transmisibles más reportadas por migrantes venezolanos son la malaria, el VIH/SIDA y las hepatitis virales. Para el 31 de julio de 2019 el Sistema Nacional de Vigilancia en Salud Publica SIVIGILA reportó que la malaria fue el evento de salud pública con el mayor número de migrantes venezolanos notificados (1 265 casos), lo cual significó el 22,8\% de las notificaciones hechas sobre esta población. El VIH representó el 6,4\% de las notificaciones (357 casos); y las hepatitis virales, teniendo en cuenta hepatitis A, $\mathrm{B}, \mathrm{C}$ y D, representaron el $2,7 \%$ de las notificaciones $(156 \text { casos })^{8}$. 
Lo anterior obedece a que tanto Venezuela como Colombia tienen regiones endémicas de malaria. Además, a pesar de que la malaria es una enfermedad prevenible y tratable, en poblaciones en condiciones vulnerables continúa generando afectaciones, en particular entre migrantes caminantes que se exponen a picaduras del mosquito vector, condiciones selváticas durante el cambio de un lugar a otro e insuficiente uso de insecticidas y toldillos?. Las hepatitis virales son prevenibles con inmunización (Hepatitis B - VHB), medicamentos (Hepatitis C - VHC) y alimentos y agua seguros; no obstante, los migrantes y refugiados no tienen un fácil acceso a esquemas de vacunación, a medicamentos innovadores, programas de tamizaje e identificación de factores de riesgo y a alimentos debidamente manipulados ${ }^{10,11}$. Finalmente, las personas migrantes se encuentran particularmente expuestas a la explotación sexual, trata de personas y al sexo como supervivencia ${ }^{12}$. Las mujeres y niñas migrantes que viajan sin redes de apoyo son susceptibles a estos riesgos, por lo cual son más vulnerables a adquirir infecciones de trasmisión sexual como el $\mathrm{VIH}^{12}$.

Teniendo en cuenta estos escenarios, nuestra pregunta de investigación es ¿cómo los migrantes venezolanos en Colombia utilizan los servicios de salud para atender enfermedades infecciosas? Para responder esta pregunta, es necesario analizar el uso de los servicios de salud por parte de la población migrante y refugiada venezolana en Colombia, específicamente por los diagnósticos de malaria, hepatitis virales y VIH/SIDA durante 2018 y 2019.

\section{Metodología}

Estudio con una metodología mixta que combinó análisis cualitativo y cuantitativo. El análisis cualitativo se basó en 12 grupos focales de discusión en seis ciudades con una alta presencia de personas migrantes y refugiadas venezolanas en 2019: Bogotá (352 431 migrantes); Cúcuta (104 981); Barranquilla (99 251); Riohacha (54 536); Cartagena (52 486) y Santa Marta (43 129)5. En cada ciudad se realizaron dos grupos focales, uno con mujeres y otro con hombres. En total 153 personas participaron (79 hombres y 74 mujeres). Los grupos focales de discusión permitieron identificar experiencias al momento de utilizar los servicios de salud a partir de las siguientes categorías: necesidades en salud, barreras de acceso a los servicios de salud, cobertura universal en salud, y desenlaces, experiencias y expectativas sobre los servicios de salud. La información fue codificada y analizada en el software N-Vivo.
El análisis cuantitativo se basó en consultas del cubo de atenciones en salud del Ministerio de Salud y Protección Social (MSPS) a través de la Bodega de Datos del Sistema Integrado de la Protección Social (SISPRO). En la Tabla 1 se encuentran los códigos de los diagnósticos de malaria, hepatitis virales y VIH/ SIDA que fueron seleccionados según la Clasificación Internacional de Enfermedades -CIE 10.

Tabla 1. Códigos CIE 10 utilizados para la búsqueda de reportes de malaria, hepatitis virales y VIH/Sida.

\begin{tabular}{ccc}
\hline Malaria & VIH/Sida & Hepatitis virales \\
\hline B500-B54X & B20-B24 & B15-B19 \\
\hline
\end{tabular}

Fuente: Dirección de investigaciones. Asociación Profamilia.

Atendiendo los requerimientos de la circular 000029 del Ministerio de Salud y Protección Social de 2017 sobre la oferta y acceso a los servicios de salud de la población migrante ${ }^{13}$, la consulta en RIPS se realizó a través del CUBO SISPRO Circular 029, filtrando las siguientes categorías: país, tipo de diagnóstico, tipo de evento RIPS, año de atención, prestador geografía y sexo. Al obtener los datos completos de 2018 y 2019 fue posible identificar el porcentaje de variación del uso de servicios de salud por parte de población migrante y refugiada venezolana. Esto permitió realizar un análisis comparativo en las cifras de los dos años priorizados, identificando el número de personas migrantes y refugiadas atendidas para cada una de las enfermedades transmisibles que se discuten en el artículo. Además, al desagregar los datos de cada enfermedad y cada año por sexo, fue posible evidenciar, según sexo, cuál ha tenido el mayor uso de servicios y cuál tuvo el mayor aumento de un año a otro. En el caso específico de las hepatitis virales se realizó una búsqueda desagregada por los distintos tipos de hepatitis a nivel nacional para conocer el comportamiento del uso de servicios de acuerdo a cada tipo.

\section{Triangulación de resultados}

Los resultados cualitativos y cuantitativos fueron triangulados a través del contraste de las percepciones y experiencias encontradas sobre el acceso a los servicios de salud con los datos del uso de servicios por eventos de enfermedades infecciosas. Esto permitió identificar barreras y necesidades asociadas a la atención en salud que influyen y son transversales en el acceso a tratamiento de enfermedades infecciosas por parte de la población migrante en Colombia. La Figura 1 hace una síntesis del proceso de triangulación de resultados. 


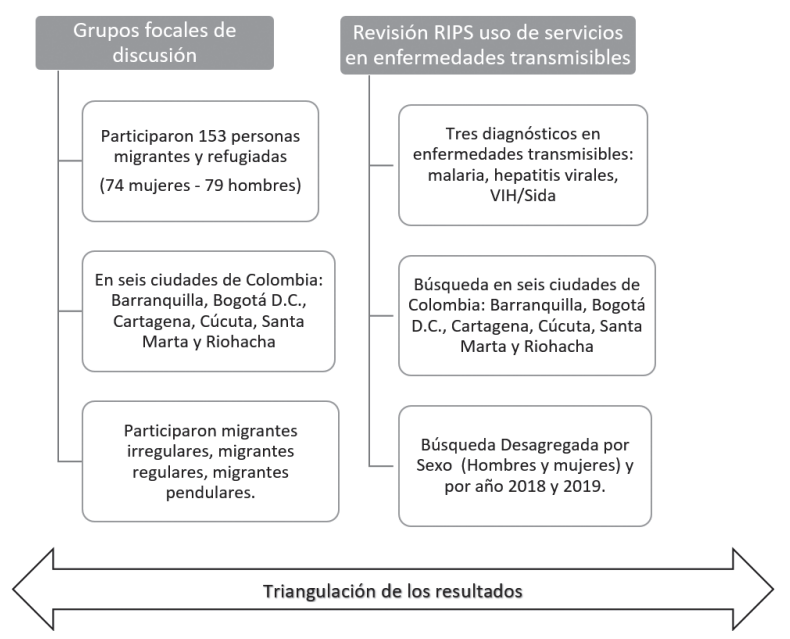

Figura 1. Síntesis método de investigación.

\section{Resultados}

Los resultados de la investigación se presentan de la siguiente forma: i) experiencias del uso de servicios de salud de las personas migrantes y refugiadas venezolanas en Colombia y ii) uso de servicios de salud por enfermedades infecciosas por parte de esta población.

\section{Experiencias del uso de servicios de salud de la población migrante $y$ refugiada venezolana en Colombia}

A continuación se relacionan las principales barreras que experimenta la población migrante y refugiada venezolana en Colombia al momento de acceder a los servicios de salud:

\section{Falta de aseguramiento en salud}

En los grupos focales realizados, los participantes estuvieron de acuerdo con que una de las principales limitaciones para acceder a servicios de salud en Colombia tiene que ver con la falta del permiso especial de permanencia (PEP). Debido a que este documento significa permanecer en el país de manera legal, aquella persona que lo porte podrá acceder a los servicios dispuestos para la población colombiana, pero aquella que no, será considerada como migrante irregular.

Esta diferenciación pone en un mayor grado de vulnerabilidad a los migrantes en situación irregular, ya que al no contar con el PEP no tienen oportunidad de afilarse al sistema de salud colombiano y, por lo tanto, la atención que reciben se reduce al servicio de urgencias: Aqui el que no tiene el PEP no lo atienden en salud, ahi te puedes quedar muriéndote, te mueres porque mientras no tengas. Incluso, los que tienen el PEP sino se está en el SISBEN, tampoco te atienden, aqui te piden el SISBEN, si no tienes SISBEN no te atienden.

Participante - Grupo focal de mujeres en Barranquilla.

Como lo muestra la cita, este problema no es exclusivo de los migrantes en situación irregular, ya que en Colombia aquellos que cuentan con PEP, también han experimentado dificultades para la afiliación al sistema de salud. Por lo general los migrantes regulares e irregulares aseguraron sentirse confundidos por los trámites burocráticos $\mathrm{y}$ administrativos que se convierten en barreras de acceso dentro del proceso de aseguramiento en salud:

Ha pasado en ocasiones que uno se tiene que resignar, fui para allá y no me lo recibieron, me hicieron el papel, otro papel, entonces para acá, otro papel, entonces eso se, uno lo toma como mamadera de gallo, de aqui para allá de allá para acá, entonces, eso es pasaje, para cualquier sitio es pasaje que sale de tu bolsillo, uno se resigna que no lo van a atender.

Participante - Grupo focal con hombres en Cartagena

La inversión de tiempo, el gasto de bolsillo y los trámites burocráticos para la afiliación en salud, hacen que las personas migrantes y refugiadas prefieran dejar esto de lado y prioricen otras necesidades que consideran más urgentes como la búsqueda de oportunidades laborales y el cuidado de las personas a cargo.

\section{Falta de información sobre el sistema de salud colombiano}

Debido a las diferencias entre el sistema de salud colombiano y el venezolano las personas migrantes y refugiadas afirmaron desconocer o tener inquietudes sobre los mecanismos de aseguramiento y prestación de servicios dentro del Sistema General de Seguridad Social en Salud (SGSSS). Migrantes y refugiados reconocieron no haber tenido la posibilidad de acceder a información clara y oportuna que permita resolver inquietudes específicas relacionadas con el sistema de salud colombiano. 
-¿Ustedes conocen sus derechos frente al acceso en atención de salud en Colombia?

$-N o$.

-Nada.

-No, yo no puedo decir, es que tal cosa, porque no conozco nada.

-El tema de salud se desconoce.

-Lo que pasa es que nosotros nos hemos acostumbrado a una cosa que no tratamos de exigir para que no nos saquen las uñas, no nos saquen los ojos.

-Preferimos no hablar.

Participante - Grupo focal con mujeres en Santa Marta

La desinformación no emergió como un desafío exclusivo de las personas migrantes que no han logrado afiliarse al sistema de salud. En los grupos focales los migrantes que contaban con afiliación al SISBEN aseguraron tener dudas sobre la cobertura de su aseguramiento, pues desconocían si al moverse entre los departamentos del país este los cubriría en todo momento.

\section{Apropiación insuficiente de los lineamientos de gobierno para la atención en salud de la población migrante y refugiada venezolana}

La falta de información sobre el funcionamiento del sistema de salud colombiano deriva en que las personas migrantes y refugiadas tengan dudas sobre sus derechos en salud en Colombia. Los grupos focales permitieron identificar que la población migrante no tiene claro cómo puede acceder a los servicios de urgencias, cómo se determina una urgencia y en qué lugares se prestan estos servicios:

- ¿Ustedes conocen puntualmente cuáles son sus derechos frente al acceso de servicios de salud en Colombia?

- No.

-Bueno, los únicos derechos que ellos [los prestadores] dicen que por emergencia entra y si no es emergencia no entra.

- El detalle es saber a quién llamar en una emergencia [...] y que aqui determinen qué es una emergencia, porque si yo voy por dolor de cabeza, porque no puedo ver, voy a desmayarme, estoy vomitando, para mí eso es una emergencia.

Participantes - Grupo focal de mujeres en Bogotá. Según la sentencia unificada 677 del 2017 de la Corte
Constitucional la atención de urgencias se debe prestar sin solicitar documentos o pagos de los servicios para generar acceso ${ }^{7}$. Sin embargo, algunas personas migrantes y refugiadas aseguraron que les han negado los servicios de urgencias por no tener el PEP o estar afiliados al sistema de salud, además han tenido que cubrir los costos de las atenciones. Esto evidencia la falta de apropiación de los lineamientos de atención en salud a población migrante en Colombia por parte de las IPS y la falta de información de los prestadores de servicios al momento de atender a esta población.

-A mí no me atendieron los primeros días, cuando tenía paludismo.

- ¿Ah, a usted le dio paludismo acá en Colombia?

-Sí, no me atendían en ningún lado.

- ¿Y cuál era la razón, que le decían, por qué no?

-No, no, deben tener el seguro, no, nosotros somos venezolanos, no, no puede. Hasta que llegue mal, mal, que ya no podía caminar y él me llevó y cuando me vieron, ah, que tiene usted, paludismo, me vieron de una vez, para dentro para el hospital.

Participante - Grupo focal de hombres en Bogotá

Los obstáculos hasta acá señalados derivan en riesgos para la salud de las personas migrantes y refugiadas. La falta de garantía de acceso a promoción, prevención y detección temprana de enfermedades conlleva una serie de amenazas para el bienestar de migrantes y refugiados, entre ellas el riesgo de sufrir graves afectaciones por esperar a que los síntomas sean considerados como una urgencia. La aparición de enfermedades infecciosas para migrantes y refugiados puede tener desenlaces fatales si las personas continúan sin acceso oportuno y de calidad en servicios de salud.

Uso de servicios de salud de la población migrante y refugiada venezolana en Colombia por enfermedades infecciosas

A continuación, en la Tabla 2 se presentan los resultados encontrados a partir de la revisión del número de personas migrantes atendidas en Colombia por malaria, hepatitis virales y VIH/SIDA, entre 2018 y 2019 , desagregados por sexo en las seis ciudades priorizadas. 
Tabla 2. Número de migrantes y refugiados venezolanos atendidos por malaria, hepatitis virales y VIH/Sida en seis ciudades de Colombia, según sexo y año.

\begin{tabular}{|c|c|c|c|c|c|c|c|c|c|c|}
\hline \multirow{2}{*}{ Año } & \multirow{2}{*}{$\frac{\text { Diagnóstico }}{\text { Ciudad }}$} & \multicolumn{2}{|c|}{ Malaria } & \multicolumn{4}{|c|}{ Hepatitis virales } & \multicolumn{2}{|c|}{ VIH/Sida } & \multirow[b]{2}{*}{ Total } \\
\hline & & Femenino & Masculino & Total & Femenino & Masculino & Total & Femenino & Masculino & \\
\hline \multirow{7}{*}{$\stackrel{\infty}{\stackrel{\circ}{\circ}}$} & Barranquilla & 5 & 5 & 10 & 0 & 5 & 5 & 5 & 9 & 14 \\
\hline & Bogotá, D.C. & 10 & 12 & 22 & 14 & 24 & 38 & 39 & 212 & 251 \\
\hline & Cartagena & 0 & 0 & 0 & 2 & 2 & 4 & 1 & 0 & 1 \\
\hline & Cúcuta & 36 & 65 & 101 & 33 & 56 & 89 & 16 & 30 & 46 \\
\hline & Riohacha & 6 & 5 & 11 & 10 & 3 & 13 & 5 & 4 & 9 \\
\hline & Santa Marta & 3 & 2 & 5 & 4 & 3 & 7 & 4 & 7 & 11 \\
\hline & Nacional & 270 & 322 & 592 & 156 & 170 & 326 & 161 & 440 & 601 \\
\hline \multirow{7}{*}{$\stackrel{\curvearrowright}{\circ}$} & Barranquilla & 2 & 1 & 3 & 9 & 6 & 15 & 16 & 16 & 32 \\
\hline & Bogotá, D.C. & 23 & 41 & 64 & 23 & 42 & 65 & 107 & 387 & 494 \\
\hline & Cartagena & 3 & 2 & 5 & 2 & 8 & 10 & 11 & 9 & 20 \\
\hline & Cúcuta & 36 & 33 & 69 & 34 & 28 & 62 & 242 & 670 & 912 \\
\hline & Riohacha & 3 & 5 & 8 & 2 & 3 & 5 & 8 & 4 & 12 \\
\hline & Santa Marta & 3 & 2 & 5 & 1 & 1 & 2 & 5 & 7 & 12 \\
\hline & Nacional & 540 & 579 & 1119 & 224 & 269 & 493 & 731 & 1645 & 2376 \\
\hline $\begin{array}{l}\text { Aumer } \\
\text { nacion }\end{array}$ & $\begin{array}{l}\text { to porcentual a nivel } \\
\text { l de } 2018 \text { a } 2019\end{array}$ & $100,0 \%$ & $79,8 \%$ & $89,0 \%$ & $43,5 \%$ & $58,2 \%$ & $51,2 \%$ & $354,0 \%$ & $273,8 \%$ & $295,3 \%$ \\
\hline
\end{tabular}

Fuente: Cubo RIPS - Migrante. Sistema de Información de la Protección Social (SISPRO). Ministerio de Salud y Protección Social (MSPS) de Colombia. Consultado el 4 de abril de 2020.

Malaria: a nivel nacional fueron atendidas 592 personas migrantes venezolanas en 2018 y 1.089 en 2019, esto representa un incremento del 89\% (497 personas) de un año a otro. Además, durante los dos años se evidenció que los hombres migrantes (322 en 2018 y 579 en 2019) usaron más los servicios por malaria en comparación con las mujeres migrantes ( 270 en 2018 y 540 en 2019). Sin embargo, el mayor porcentaje de variación de un año a otro, según sexo, se presentó en las mujeres, quienes tuvieron un aumento del $100 \%$ en comparación con los hombres, en estos últimos el uso aumentó un 79,8\%.

Entre las ciudades priorizadas se encontró que para los dos años Cúcuta y Bogotá tuvieron la mayor cantidad de migrantes atendidos por malaria, respecto a las otras cuatro ciudades (Barranquilla, Cartagena, Santa Marta y Riohacha). En Cúcuta se evidencia el mayor número de personas atendidas durante los dos años y en Bogotá se presenta el mayor aumento respecto al resto de ciudades, ya que se registraron 42 casos más en 2019 respecto al 2018. La situación de Bogotá es llamativa ya que, a pesar de no ser una región endémica para malaria, en 2019 reportó el mayor número de personas migrantes y refugiadas atendidas por este evento. Esto puede explicarse porque probablemente las personas migrantes y refugiadas, principalmente caminantes que han estado en regiones endémicas del país, llegan a Bogotá con necesidades de atención por malaria que no son atendidas en los municipios recorridos durante el tránsito.

Hepatitis Virales: a nivel nacional fueron atendidas 326 personas migrantes en 2018 y 493 en 2019, lo cual representa un incremento de 51,2\% (167 personas más en 2019 en comparación al año anterior). Durante los dos años, los hombres migrantes (170 en 2018 y 269 en 2019) acudieron más a los servicios de salud por diagnósticos asociados a hepatitis virales respecto a las mujeres (156 en 2018 y 224 en 2019). Adicionalmente, en 2019 el porcentaje de aumento de hombres atendidos $(58,2 \%)$ fue superior al de las mujeres $(43,5 \%)$.

Entre las ciudades analizadas Cúcuta evidenció el mayor número de personas migrantes y refugiadas atendidas por hepatitis viral, seguida de Bogotá. La variación de un año a otro fue mayor en Cartagena, Bogotá y Barranquilla. En contraposición Cúcuta, Riohacha y Santa Marta evidenciaron una reducción en el número de población migrante y refugiada atendida por casos de hepatitis virales.

Teniendo en cuenta que en el grupo de las hepatitis virales se encuentran diversos tipos de hepatitis, un análisis de los datos a nivel nacional permitió evidenciar que el tipo A es el evento por el cual un mayor número 
de personas migrantes han accedido a servicios de salud en Colombia (145 personas (44,4\%) en 2018 y 195 personas (39,5\%) en 2019). Por su parte, otras hepatitis como la tipo B (28 (8,5\%) en 2018 y $33(6,6 \%)$ en 2019$)$ y la tipo C (4 (1,2\%) en 2018 y $8(1,6 \%)$ en 2019$)$ tienen menor frecuencia en el uso de los servicios de salud de la población migrante en Colombia.

VIH/SIDA: en Colombia durante el 2018 fueron atendidas 601 personas migrantes venezolanas por VIH/SIDA, mientras que en 2019 se reportaron 2 376, esto representa un aumento de 295,3\% (1 775 personas más en 2019 con respecto al año anterior). Según sexo, los hombres migrantes han sido quienes más han buscado los servicios de salud para solicitar atención por VIH/SIDA tanto en 2018 (440) como en 2019 (1 645). No obstante, el porcentaje de aumento de personas atendidas de 2018 a 2019 es mayor en las mujeres (354\%) en comparación con el de los hombres $(273,8 \%)$.

Entre las ciudades analizadas Cúcuta y Bogotá registraron el mayor número de migrantes atendidos por VIH/SIDA tanto en 2018 como en 2019, en comparación con el resto de ciudades. Igualmente, Cúcuta y Bogotá fueron las ciudades con el mayor número de aumento de un año a otro. Es importante mencionar que, aunque en menor proporción, Barranquilla, Cartagena, Santa Marta y Riohacha también tuvieron un aumento en el número de personas atendidas de un año a otro.

\section{Discusión}

Este estudio contribuye con nueva evidencia sobre el uso de servicios de salud por enfermedades infecciosas en población migrante venezolana en Colombia y describe la forma en que las personas migrantes y refugiadas se enfrentan a barreras y necesidades que dificultan su acceso a servicios de salud. Estas barreras tienen implicaciones directas en el manejo y control de las enfermedades infecciosas, ya que las personas migrantes al no contar con aseguramiento y afiliación al sistema de salud, no tienen la posibilidad de identificar a tiempo y tratar enfermedades como la malaria, las hepatitis virales y el VIH/SIDA. Este escenario no solo agrava la severidad sino que también puede aumentar la prevalencia de enfermedades infecciosas en esta población, acentuando las necesidades en materia de acceso a salud

El aumento en el número de migrantes atendidos por VIH/SIDA, y en menor medida por malaria y hepatitis virales, hace referencia a las múltiples necesidades que la población migrante venezolana está afrontando en Colombia, ya que refleja la falta de acciones de prevención y control para reducir las enfermedades infecciosas. En este sentido, las cifras de personas migrantes atendidas por estas enfermedades hablan de las condiciones de vida de los migrantes en Colombia.

El aumento en el número de personas migrantes atendidas por malaria en Bogotá, ciudad que no es considerada como territorio endémico para malaria, sugiere que probablemente los migrantes y refugiados contraen la enfermedad durante su proceso migratorio y enfrentan dificultades para acceder a elementos que eviten el contagio, como repelentes y toldillos. Además, esto evidencia que al contraer la enfermedad durante el recorrido por regiones endémicas, los migrantes y refugiados no logran tener acceso a servicios de salud para atender su condición de forma oportuna.

El mayor número de personas migrantes atendidas por hepatitis virales en Bogotá y Cúcuta revela la necesidad de definir estrategias que garanticen para los migrantes y las comunidades que los acogen el acceso a tamizaje, inmunización y a alimentos y aguas en condiciones de salubridad. La falta de estas medidas de prevención durante los procesos de migración de las personas venezolanas en Colombia explica por qué, durante 2018 y 2019, la mayoría de los migrantes han accedido a servicios de salud por casos, principalmente, de hepatitis de tipo A.

Frente a los casos de VIH/SIDA, el número de personas migrantes atendidas aumentó en Cúcuta y Bogotá, lo cual, además de relacionarse con el mayor número de migrantes en las dos ciudades, indica un mayor flujo de población migrante con VIH hacia Cúcuta y Bogotá en busca de atención. No obstante, pese a este incremento en el número de personas migrantes atendidas por $\mathrm{VIH} /$ SIDA, no se puede afirmar que el país esté sufriendo una emergencia de esta enfermedad producto de la migración venezolana.

En 2018 el Instituto Nacional de Salud (INS) afirmó que en Colombia el VIH/SIDA viene en aumento progresivo desde el 2008: la incidencia de VIH/SIDA fue de 13,7 casos por cada 100.000 habitantes en 2008 y, diez años más tarde (2018) fue de 28,1 casos por cada 100.000 habitantes ${ }^{14,15}$. Este escenario deja en evidencia que en Colombia el incremento en los casos de VIH/SIDA se dio mucho antes que iniciara la migración masiva de población venezolana. Además, no hay evidencia en la literatura que permita identificar el número de migrantes venezolanos que salieron de su país viviendo con VIH/SIDA ${ }^{16}$. 
En este sentido, las nociones que vinculan a los migrantes con la diseminación de enfermedades infecciosas en los países de acogida no tienen en cuenta las condiciones en las que esta población vive durante su proceso de migración y las barreras que enfrentan para acceder a servicios de salud de manera oportuna, reforzando el estereotipo del migrante como un agente externo que aumenta las probabilidades de propagar enfermedades ${ }^{17}$. Así, las enfermedades infecciosas más allá de ser 'enfermedades importadas' por los migrantes a los países de acogida, son riesgos a los que se enfrenta esta población durante sus desplazamientos.

Las personas venezolanas en Colombia presentan múltiples barreras a la hora de acceder a los servicios de salud, las cuales explican por qué han aumentado las necesidades de esta población migrante en recibir atención por eventos de malaria, hepatitis virales y VIH/SIDA. Esto requiere, primero, reformas a nivel nacional que flexibilicen el acceso de las personas migrantes y refugiadas, es decir que el acceso no se restrinja a servicios de urgencias. Segundo, es necesario una respuesta oportuna por parte de las entidades de servicios de salud territoriales, pues estas tienen a su cargo el financiamiento de los prestadores de servicios, y por ende, las atenciones a la población migrante y refugiada a nivel local en los territorios. Al garantizar atención oportuna y de calidad será posible mitigar y controlar el aumento en casos de enfermedades infecciosas en esta población.

Además, a partir de las experiencias de la población migrante al momento de buscar acceso a salud en Colombia, es clave afirmar, que a nivel local es necesario generar una mayor apropiación de los lineamientos nacionales establecidos para garantizar la atención. Lo anterior incluye reconocer las necesidades y las especificidades de vida de la población migrante, como el riesgo de sufrir aislamiento social producto de la xenofobia y la discriminación ${ }^{4}$, o el riesgo de contraer enfermedades transmisibles debido a procesos de trata de personas $^{18}$, falta de redes de apoyo, falta de acceso a métodos anticonceptivos y violencias de género ${ }^{19}$, por lo cual es importante tener en cuenta que se evidencia un mayor aumento en el número de mujeres migrantes atendidas por enfermedades transmisibles entre 2018 y 2019 en comparación a los hombres.

Para finalizar, vale la pena resaltar que los servicios de urgencias a los cuales tienen acceso las personas migrantes venezolanas, no son suficientes para generar una respuesta efectiva a casos de malaria, hepatitis virales y VIH/SIDA, pues lo aquí presentado revela que los migrantes necesitan acceso a aseguramiento en salud y a atención primaria, desde la cual puedan recibir pruebas diagnósticas y medicamentos indicados para tratar de manera oportuna eventos de enfermedades transmisibles.

\section{Recomendaciones}

Para Colombia, la migración de población venezolana, debe ser comprendida como una oportunidad para mejorar la respuesta del sistema de salud a enfermedades como las transmisibles. Es importante que instituciones de gobierno y tomadores de decisiones, vean en la migración, la posibilidad para hacer más evidentes los problemas de acceso o cobertura del servicio de salud y así coordinar respuestas que no solo estén enfocadas en la población migrante y refugiada sino también en la población de acogida. Por lo anterior, a partir de la evidencia generada, es posible plantear las siguientes recomendaciones a las autoridades de salud tanto nacionales como locales, prestadores de servicios, instituciones de respuesta humanitaria, entre otros:

1. Identificar procesos que faciliten la efectividad de programas de prevención, control y manejo de enfermedades como las hepatitis virales, la malaria y el VIH/Sida.

2. Asegurar el acceso de la población migrante y refugiada a atención primaria en salud. Esto incluye acceso a tamizaje y tratamientos oportunos.

3. Reforzar programas de tamizaje existentes a nivel local, territorial y nacional, en los cuales se incluya elementos de identificación de poblaciones en riesgo, inicio de tratamiento, monitoreo $\mathrm{y}$ evaluación de casos.

4. Promover campañas de prevención, manejo y control de enfermedades infecciosas en migrantes y refugiados, así como, con población colombiana más vulnerable.

5. Promover uso de métodos anticonceptivos de barrera, incluso cuando se está haciendo uso de otros métodos anticonceptivos.

6. Mejorar sistemas de referencia y contra referencia y aprovechar el desarrollo del sistema de vigilancia en salud pública a nivel local.

7. Garantizar que los servicios de salud dispuestos para la población migrante estén libres de violencias de género y xenofobia, promoviendo la respuesta a las necesidades y especificidades de la población migrante y refugiada venezolana en el país. 


\section{Limitaciones del estudio}

En el análisis de datos cuantitativos, se debe tener en cuenta que hay un subregistro de aquellas personas migrantes que no lograron recibir atención y, por lo tanto, no se encuentran en la plataforma de SISPRO. Además, que algunas instituciones prestadoras de salud aunque han prestado atención en salud a migrantes no hicieron el registro en el RIPS en las fechas correspondientes. De igual forma, se debe considerar que los grupos focales no representan la realidad de todas las personas migrantes ubicadas en las ciudades priorizadas, ya que se refieren a las experiencias de quienes aceptaron participar en la investigación.

\section{Agradecimientos}

Esta investigación se llevó a cabo gracias a las personas migrantes y refugiadas venezolanas que accedieron a participar en los grupos focales en las seis ciudades priorizadas. De igual manera, agradecemos a Daniela Roldan por sus comentarios y revisión crítica a una primera versión del artículo.

Apoyos recibidos: La investigación fue financiada por la Oficina de los Estados Unidos de Asistencia para Desastres en el Extranjero (OFDA - USAID) y la Asociación Profamilia.

\section{Consideraciones éticas}

La investigación fue aprobada por el comité de ética de Profamilia el 22 de octubre del 2019 mediante el oficio CEIP-2019-19.

\section{Conflicto de interés}

Los autores declaran no tener conflicto de intereses y que los puntos de vista expresados son responsabilidad de los autores y no de las instituciones en las que trabajan.

\section{Referencias}

1. Roca C, De Balanzó X. Enfermedades importadas en inmigrantes: Mito y realidad. Anales del Sistema San Navarra. 2006; 29(Supl 1): 139-144. doi: $10.4321 / \mathrm{S} 1137-66272006000200012$

2. OIM. Informe sobre las migraciones en el mundo 2020. Ginebra; 2019. https://publications.iom.int/ system/files/pdf/wmr_2020_es_0.pdf
3. OMS. Los migrantes y los refugiados presentan un mayor riesgo de tener mala salud que las poblaciones de acogida. 2019. https://www.who.int/ es/news-room/detail/21-01-2019-21-01-2019-2101-2019-migrants-and-refugees-at-higher-risk-ofdeveloping-ill-health

4. Balcells ME, Alarcón V. Enfermedades Infecciosas y migración. Una responsabilidad compartida. ARS MEDICA. 2017; 42(2): 4-6. doi: http://dx.doi. org/10.11565/arsme

5. Migración Colombia. Total de Venezolanos en Colombia corte a 31 de octubre de 2019. Colombia. 2019. https://www.migracioncolombia. gov.co/infografias/231-infografias-2019/total-devenezolanos-en-colombia-corte-a-31-de-octubrede-2019

6. MSPS. Plan de Respuesta del Sector Salud al Fenomeno Migratorio. Bogotá. 2019. https://www. minsalud.gov.co/sites/rid/Lists/BibliotecaDigital/ RIDE/DE/COM/plan-respuesta-salud-migrantes. pdf

7. Corte Constitucional. Setencia SU677/17. Colombia. 2017. https://www.corteconstitucional. gov.co/relatoria/2017/SU677-17.htm

8. MSPS. Seguimiento de la situación en salud de la población migrante procedente de Venezuela, para el período comprendido entre el 1 de marzo de 2017 al 31 de julio 2019. Colombia. 2019. https://www. minsalud.gov.co/sites/rid/Lists/BibliotecaDigital/ RIDE/VS/ED/GCFI/informe-cicular-029-cortejulio.pdf

9. OMS. Estrategia Técnica Mundial contra la Malaria. Ginebra. 2015.

10. OMS. Hepatitis viral. https://www.who.int/hiv/ topics/hepatitis/hepatitisinfo/es/

11. Sharma S, Carballo M, Feld JJ, Janssen HLA. Immigration and viral hepatitis. J Hepatol. 2015; 63: 515-522. doi: https://doi.org/10.1016/j. jhep.2015.04.026

12. Clacso. Voces y experiencias de la niñez y la adolescencia venezolana migrante en Brasil, Colombia, Ecuador y Perú. Buenos Aires. 2020.

13. MSPS. Circular externa N.000029. Colombia. 2017. https://www.minsalud.gov.co/Normatividad Nuevo/Circular No. 029 de 2017.pdf

14. INS. Boletin Epidemiológico Semanal. Comportamiento del VIH/SIDA. Bogotá; 2018.

15. CAC. Situación del VIH Sida en Colombia. Bogotá. 2019.

16. Silva Santiesteban A. Diagnostico rápido: Situación de los migrantes venezolanos con VIH en el Perú. ONUSIDA. Perú. 2019. 
17. Infante $\mathrm{C}$, Leyva R, Caballero M, Guerrero C, Cuadra $\mathrm{S}$, Bronfman M. VIH/SIDA y rechazo a migrantes en contextos fronterizos. Migr Desarrollo. 2004; 3: 45-53. doi: 10.35533/myd.0203.ci.rl.mc.cg.sc.mb

18. Observatorio Nacional de Trata de Personas. Migración Venezolana y la Trata de Personas. MinInterior. Colombia. 2018.

19. Profamilia. Evaluación de las necesidades insatisfechas en salud sexual y reproductiva de la población migrante venezolana en cuatro ciudades de la frontera colombo - venezolana. Colombia. 2018. 